\title{
Research on Quality Grade Classification Method of Daily-use Porcelain Based on Multi-agent Technology
}

\author{
Yong-Hong ZHU, Liang-Ying LI, Jun-Xiang WANG, Dong WANG \\ School of Mechanical and Electronic Engineering, Jingdezhen Ceramic Institute \\ Jingdezhen, China,zyh_patrick@163.com,Liangyingli@163.com,wjx851113851113@163.com,79973351@qq.com
}

\begin{abstract}
Due to the influence of factors such as environment, materials and so on in the ceramic production process, different levels and different kinds of defects such as crack, fall slag, deformation, roast flower sticky glaze and so on appeared on ceramic products. In this case, the artificial discerning and classification efficiency is very low. In order to improve ceramic products quality classification efficiency and intelligent level, a kind of method of applying multi-agent technology to ceramic products quality grade classification is presented, and the ceramic classification system structure and function implementation scheme based on multi-agent technology are also given in the paper. At the same time, Kalman filtering algorithm and C4.5 classification algorithm is effectively fused to process data by the multi-agent. In the end, an application example is given. Through applying the method proposed to the sampling daily-use porcelain classification, the result shows that the method proposed is effective and feasible in the daily-use porcelain classification. This will provide a new approach and new idea for the application of multi-agent technology to the ceramics field.
\end{abstract}

\section{Introduction}

In ceramic production process, uncertain factors such as raw materials preparation, temperature control and so on can lead to various defects of eventually fired ceramics, including crack, deformation, fall slag, roast flower sticky glaze, crack glaze, brown eye (pinhole, pore), spots, dirty marks, dirty A12O3, glaze peels edge peeling, excess glaze, orange glaze, glaze bubble, glazed, ripple, smoked, off color, melt hole, body powder (sludge, glaze slag), concave and convex, and so on[1]. After ceramic products out of ceramic kiln cool, all products need be classified. Unqualified products are usually removed, some products with no very serious defects are screened, and defective products which can be normally used are sold at a low price. At present, ceramic products mainly rely on manual screening, and qualified or unqualified products rely on artificial experience judgment. Hence, this kind of classification method greatly affects the production efficiency. Under the great environment of today's highly developed science and technology and acceleration of society pace, enterprises will have to keep up with the rhythm and shorten the production cycle in order to survive.

Multi-agent system is a hot research content of artificial intelligence field, starting from the mid-1980-s. In recent years, multi-agent technology research has entered the stage of relative prosperity and also slowly began to be applied in various industry fields. In complex control system, L. L. Li, etc. proposed a kind of control method of city commercial user pricing system based on multi-agent technology, and constructed corresponding agent by simulating government, users, companies, monitoring, interactive personnel and so on respectively, then used agent interactive to simulate price fluctuations led to by city gas network demand and operational changes[2] . X. M. Luo, etc. applied the multiagent technology to missile combat system, and puts forward the anti-radiation missile operational effectiveness assessment model based on Bayesian network[3]. In image processing, W. N. Xie, etc. put forward to detect and analyze the synthetic aperture radar images by using multiagent two-dimensional Otsu method, and used multi-agent genetic algorithm to determine the maximum threshold of the distance measurement function between variable and constant in order to obtain detection results[4]. However, the application of multi-agent technology to daily-use porcelain quality grade classification has not yet been reported. So far, daily-use porcelain has still be classified by manual operation. Hence, labor intensity of workers is great and production efficiency is low. Based on the above research results, the application of multi-agent technology to ceramics quality grade classification will be conducive to greatly reduce labor intensity and to improve production efficiency, and is with the vital significance.

Ceramic products quality classification in ceramic industry production is a relatively complicated process. In 
this paper, multi-agent alliance network structure was used to analyze data, a small multi-agent alliance network used for detection and classification is rebuilt on the basis of the fully multi-agent network structure of ceramic production process control system[5-8]. When the multi-agent alliance network detects and classifies ceramic products, an agent begins to interact with other agents. After the completion of interaction, through using pre-defined criteria, the interaction with the agents which generate positive impact is enhanced and the interaction with the agents which generate negative impact is abated so as to improve the automation level, to bring more profits for enterprises and to promote the development of ceramic industry.

\section{$2 \quad$ Multi-agent Technology}

Agent is a small branch of artificial intelligence. It is generally regarded that the agent concept came from the Dartmouth conference launched together by the father of artificial intelligence, a computer scientist Marvin Ming and the founder of framework theory, J. McCarthy in 1956. With the development of science and technology, the agent is given more and more new features and is generally with three basic features shown in Figure1[9]. Autonomy means that it is without persons or other devices' direct interference and with the ability to control their own behavior; Groups means that it can work with other agents together to achieve shared goals; Self-adaption means that it is with the ability to change their behavior by learning. An agent system includes four dimensional connotations such as agent, environment, interaction and organization shown in Figure2 [9]. The agent means the internal inference. The environment means that perception and action of agents in the environment. The interaction means the interaction of an agent with other agents. The organization means the structuralization and management of relationship among agents.

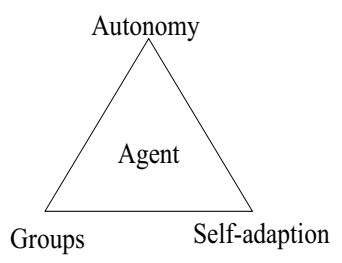

Figure 1. Three characteristics

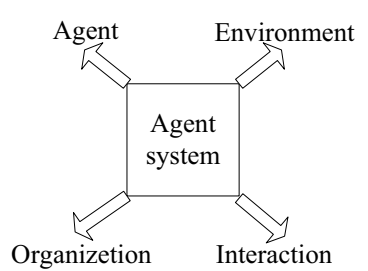

Figure 2. Four connotations
The multi-agent network structure given in the article includes complete network structure and alliance network structure shown in Figure 3 and Figure 4 respectively. Alliance network system structure-based the multi-agent system is with three-tier multi-level structure such as low layer agent, middle layer agent and high layer agent. High layer agent understands the structures of all middle layer agents and low layer agents, often interacts with the other high layer agents and manage middle layer agents at various places. Middle-layer agent manages several low layer agents, and only belongs to a high layer agent. Low layer agent is reactive agent and generally with several special functions, and only belongs a middle layer agent. Its basic components is shown in Figure 5.

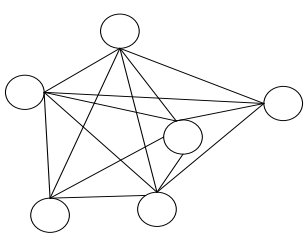

Figure. 3 Complete network Structure

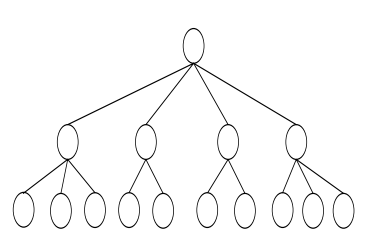

Figure. 4 Alliance network structure

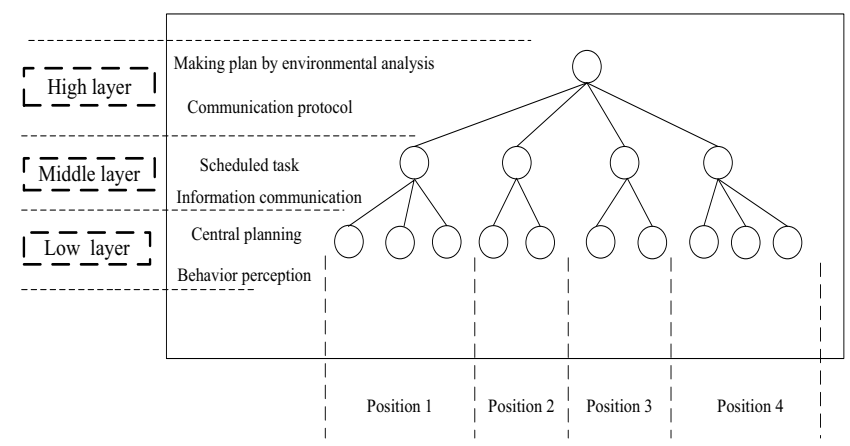

Figure 5. Composition relationship of multi-agent platform

\section{The Structural Composition and Function Modules of Daily-use Porcelains Classification System}

\subsection{Multi-agent Species of the Classification System}

The multi-agent of the classification system is mainly composed of interactive agent, task agent, management agent, resource agent and execution agent. The relationship among them is shown in Figure. 6.

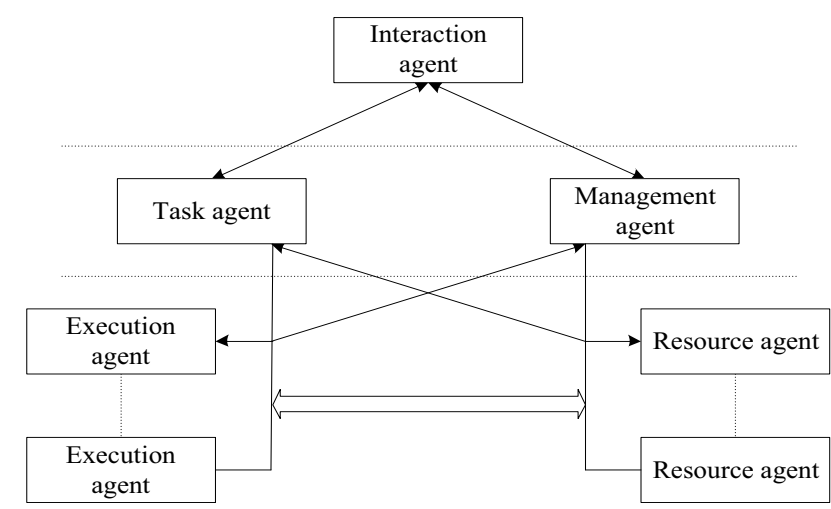

Figure 6. Multi-agent structure chart of the classification system 


\subsection{The Classification System Architecture}

Multi-agent-based daily-use porcelain quality classification system architecture is shown in Figure 7.Man-machine interface agent transforms instruction information into the system task. Then, each agent in the system completes the data analysis, data diagnosis and implementation respectively. At last, these agents feedback the results to the terminal network server and the terminal function server. The two terminal servers are data accessing terminals, but their functions is not the same in the system. In fact, there is a standby database in the system, and the information in the database can not be lost. A major role of the database is that the safe and detailed database can be used to invoke in the system if the information agent fails. Man-machine interface agent converts the final task results to man-machine interface output format, and reports the overall solution situation so as to complete final task. Finally, the users can observe the work situation of the system via the manmachine interface.

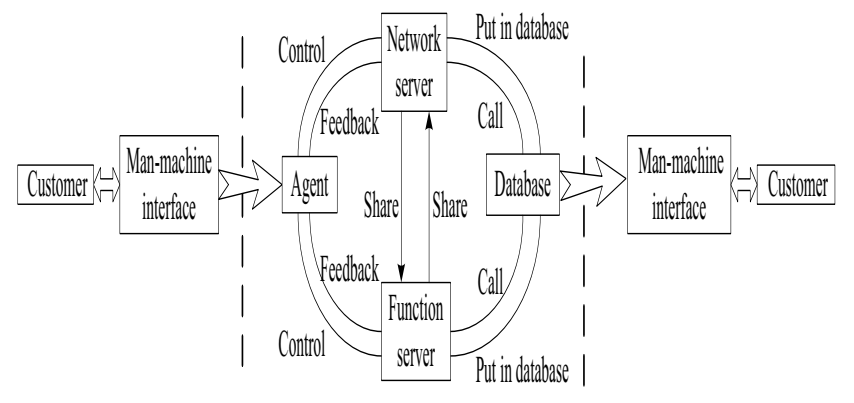

Figure 7. Multi-agent-based daily-use porcelain quality classification system architecture

\subsection{Detection and Classification System Flow}

After the completion of the whole system task allocation, the executive agent starts detection, and the resource agent starts information collection and data analysis. If the corresponding defect types and quality levels can be determined via the analysis, each agent interacts and saves useful information in order to accumulate. If there exists any abnormal, then the system further inquires and diagnoses the abnormal until the system extracts useful information and stores it in the information agent in order to invoke it at any time. The users can query the final results, including new abnormal data, defect types, the products number corresponding to quality grade, scrap percentage and so on. The detection and classification system flow chart is shown in Figure 8.

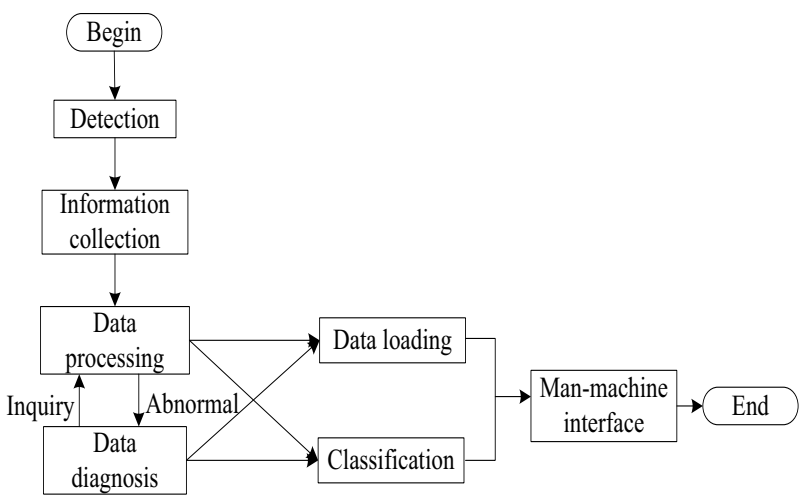

Figure 8. Detection and classification system flow chart

\subsection{The System Function Module Design}

The system is mainly used to collect and to process the information related to daily-use porcelain quality. If there are abnormal circumstances in the process, the system can give the hints and saves processed results into the database in order to query them. The system generally includes the following four modules.

User Interface Module. The module is mainly used to upload relevant detection and classification information files to the operator, and to provide the interface of querying results for the users.

Information Collection Module. The module is mainly used to collect data information related to daily-use porcelain quality, including useful information and various kinds of interference information which can not be avoided.

Information Processing Module. This module is mainly used to process the received information related to ceramic quality in accordance with the criterion defined based on multi-agent algorithms, and makes decision.

Data Access Module. The module is used to provide the relevant database access manner, so that the actuator executes tasks quickly and the users easily query the system

\subsection{Database Design}

By understanding whole production environment of dailyuse porcelain, the database of the detection and classification system includes surrounding environment information, archives information of ceramic varieties, system detection and classification tools information, national standard information of daily-use porcelain detection, detection and classification results information and so on. For instance, China standard of daily-use porcelain includes GB / T 35322009, GB 3300-91, GB-T 3295-1996, GBT 3300-2008, GBT 3301-1999, GB-T 4742-1984, GB-T 4966- 1985, GB-T 15614-1995, QBT 1503-1992 and so on. 


\section{Functions Implementation of Multi- agent System}

\subsection{Agent Functions Implementation}

Interactive Agent. Interactive agent, otherwise known as man-machine interaction agent, receives instructions from users through the man-machine interface such as defect types, defect standards and classification commands, and helps users complete the required query information in the whole production At the same time, the agent gives advice on users for the next query, and reduces the omission or repetition of the users' information query so as to improve the production efficiency.

Task Agent. Task agent receives the task instructions about ceramic defect detection and quality grade classification given by previous users. Then, it gives an overall layout of the task., and decomposes the task after the layout. At last, it assigns each agent an appropriate task through agents communication.

Management Agent. Management agent is mainly used to plan as a whole system. It monitors and feedbacks the work state of each agent by analyzing users instruction information, and coordinates each agent work by calling the knowledge base of the whole system reasonably so that they can carry out their duties and finish the operation of the whole system quickly and accurately.

Resource Agent. In this system, resource agent is connected to the terminal sensors, CCD cameras, etc. After processing the collected information, it passes processed information to the task agent and the management agent so that the management agent can arrange other agents to carry their duties reasonably and processes the data into the same space and time data understood by all agents so as to complete the data matching among agents .

Executive Agent. Executive Agent is responsible for the terminal data process and inference in the whole detection and classification system, that is, data association, state estimation, attribute estimation after detection, and controls machine classification.

Other Agents. The other agents in the system mainly refers to communication agent and information agent which play a supplementary role. When each agent communicates each other, information agent is mainly used to collect the useful information for the system so as to make the agents communicate more fluently in future. Communication agent is responsible for communicating with the other agents in the whole ceramic kiln control system and linking the union type agent information database with other agents together to share information so as to complete the whole control.

\subsection{The Implementation of Communication Among Agents}

In the production system of daily-use porcelain, the industrial equipment of the whole system is relatively fixed and control terminals are also relatively fixed. Hence, According to the above characteristics, the communication among multi agents can be realized based on Switched Ethernet. Not only Switch Ethernet switches will be isolated from the conflict in each port and avoid the spread of the conflict, but also the full bandwidth can be used for each node of access switch.

\subsection{The Implementation of Information Collection and Decision-making Process}

Man-machine interface agent translates the received instruction information into the task of the system. Task agent decomposes the task into several sub-tasks that are easy to accomplish. Management agent sends a task description information to both execution agent and resource agent and calls for tenders for each sub-task. The relevant execution agents bid for the task and the management agent determines the agent which has winned bid. The management agent sends the information to the winning bidder to confirm that it will perform the task. At the same time, it sends the information to the task agent so as to make each sub-task assigned to each successful bidder. The execution agent analyzes the task and calls the information agent and communication agent in order to get enough information for operation and reasoning, hence, each subtask has been completed. Task agent summarizes the task results of each execution agent, and get the results of the total task after comprehensive analysis. Man-machine interface agent converts the final task to the output format of man-machine interface, and reports the overall situation, hence, the task has been completed eventually. Information processing and decision-making process is shown in Figure 9.

\section{Daily-use Porcelain Classification Algorithm}

\subsection{Discrete Kalman Filtering Algorithm}

Although the $\mathrm{C} 4.5$ algorithm is with pruning function, it is very necessary for the signal data to filter in order to obtain more accurate data at the time of data processing[10]. The noise caused by light or electronic equipment can not be avoided in industrial production, At this time, not only there exists small error data, but also there exists small part of large error data. These error data had adverse effect on the detection and self-adaption of agents. These large errors which affect results are called the field value. In order to obtain the accurate and reasonable preprocessing data results, it is necessary to remove the outliers from the signal data. In this paper, the Kalman filtering algorithm is used to remove the outliers. 


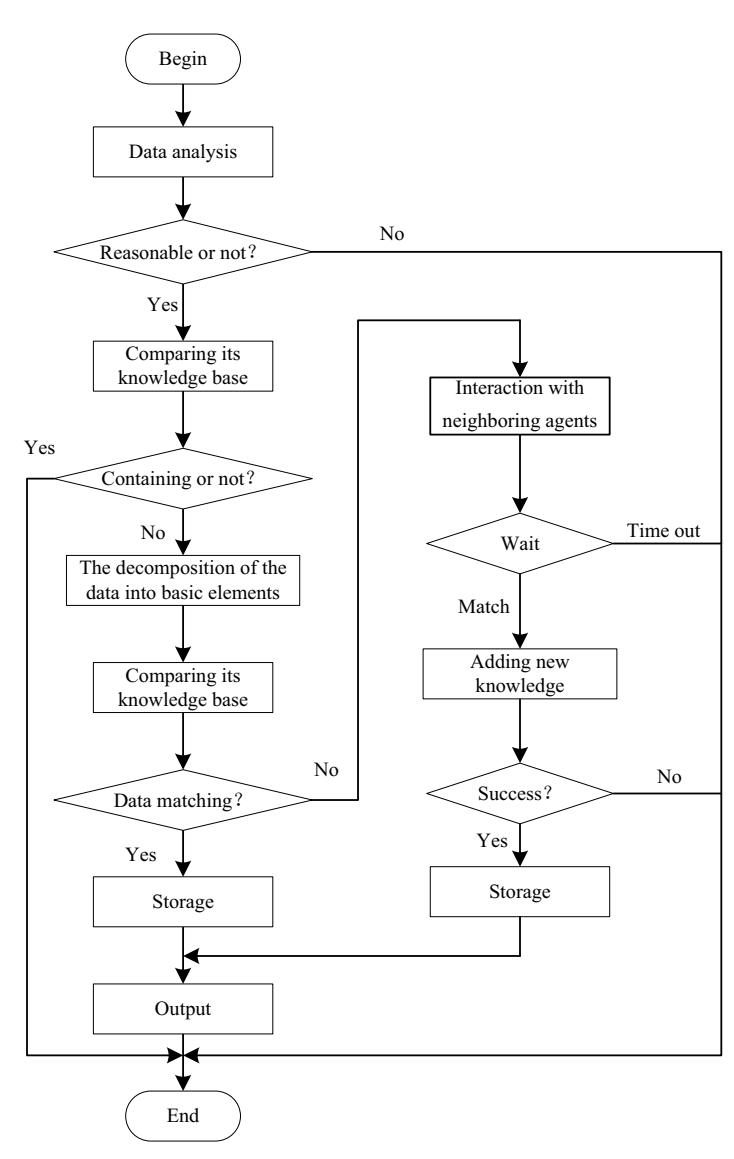

Figure 9. Information processing and decision-making process flow chart

Discrete Kalman filtering system equations.

State equation

$X(k+1)=\Phi(k+1) X(k)+G(k) w(k)$

Measurement equation

$Z(k)=H(k) X(k)+v(k)$

Where, $X(\mathrm{k}+1)$ is the state estimation vector at $t=(k+1) T, \Phi(k+1)$ is the state transfer matrix, $G(k)$ is the state noise weighting matrix, $w(k)$ is the noise vector, $Z(k)$ is the measurement vector at $t=k T, H(k)$ is the measurement transfer matrix, $v(k)$ is the measurement noise vector. $w(k)$ and $v(k)$ are uncorrelated zero mean Gaussian white noise sequence and satisfy the following equations.

$$
\left\{\begin{array}{l}
E\left[w(k) w^{T}(i)\right]=Q(k) \delta_{k i} \\
E\left[v(k) v^{T}(i)\right]=R(k) \delta_{k i} \\
E\left[w(k) v^{T}(i)\right]=0
\end{array}\right.
$$

Where, $Q(k)$ and $R(k)$ are the state noise matrix and measurement noise matrix respectively, $\delta_{k i}$ is Kronecker function with $\delta_{k i} \neq 0(k=i), \delta_{k i}=0(k \neq i)$.

Discrete Kalman Filtering Algorithm.

Filtering gain updating equation:

$$
K(k)=P(k \mid k-1) H^{T}(k) \times\left[H(k) \times P(k \mid k-1) H^{T}(k)+R(k)\right]^{-1}
$$

State updating equation:

$$
\begin{aligned}
& \hat{X}(k+1 \mid k+1)=\hat{X}(k+1 \mid k)+K(k+1) \times \\
& {[Z(k+1)-H(k+1) \hat{X}(k+1 \mid k)]}
\end{aligned}
$$

One step prediction equation of state:

$$
\hat{X}(k \mid k-1)=\Phi(k \mid k-1) \hat{X}(k-1 \mid k-1)
$$

One step prediction equation of covariance:

$$
P(k+1 \mid k)=\Phi(k+1 \mid k) P(k \mid k) \Phi^{T}(k+1 \mid k)+
$$

$$
G(k) Q(k) G^{T}(k)
$$

Covariance updating equation:

$$
P(k+1 \mid k+1)=[I-K(k+1) \times H(k+1)] P(k+1 \mid k)
$$

Through Kalman filtering algorithm, the measured value is compared with the known feature vector and weighted correction of the state estimates is made. This algorithm can be summed up as data prediction and the cycle recursive process of measurement data correction.

\subsection{C4.5 Decision Tree Classification Algorithm}

Assume that the measured data is the set A. $\left(c_{1}, c_{2}, c_{3}, \cdots\right.$ denotes $\mathrm{m}$ categories in $A$, $\left(c_{1}, c_{2}, c_{3}, \cdots \quad\right.$ denotes $\mathrm{n}$ the values of attribute $\mathrm{C}$ in $\mathrm{A}$, $\left(A_{1}, A_{2}, A_{3}, \cdots \quad\right.$ denotes $\mathrm{n}$ subsets of $A$ divided by $\mathrm{n}$ values of attribute $\mathrm{C},\left|A_{i}\right|$ denotes the sample number at 
$C=C_{i},\left|B_{j}\right|=\operatorname{freq}\left(B_{j}, A\right)$ denotes the sample number of $B_{j},\left|B_{j c}\right|$ denotes the sample number of $B_{j}$ at $C=C_{i}$. C4.5 decision tree algorithm is as follows.

Category probability. $P\left(B_{j}\right)=\left|B_{j}\right| /|A|$

Attribute probability. $P\left(c_{j}\right)=\left|A_{j}\right| /|A|$

Conditional probability of belonging to the category $B_{j}$ at $C=C_{i}$.

$P\left(B_{j} \mid C_{i}\right)=\left|B_{j c}\right| /\left|A_{i}\right|$

Class information entropy.

$H(B)=-\sum_{j} p\left(B_{j}\right) \log _{2} p\left(B_{j}\right)=\operatorname{Info}(A)$

Conditions information.

$$
\begin{aligned}
H(B / C) & =-\sum_{j} p\left(B_{j} / C_{i}\right) \log _{2} p\left(B_{j} / C_{i}\right)= \\
& -\sum_{i=1}^{n} \frac{\left|A_{i}\right|}{|A|} \operatorname{Info}\left(A_{i}\right)=\operatorname{Info}_{c}(A)
\end{aligned}
$$

Information gain.

$$
\begin{aligned}
& I(B, C)=H(B)-H(B / C)= \\
& \quad \operatorname{Info}(A)-\operatorname{Info}(A)=\operatorname{gain}(c)
\end{aligned}
$$

Splitting information.

$$
\begin{aligned}
H(C)= & -\sum_{i} p\left(C_{i}\right) \log _{2} p\left(C_{i}\right)= \\
& -\sum_{i=1}^{n} \frac{\left|A_{i}\right|}{|A|} \log _{2} \frac{\left|A_{i}\right|}{|A|}=\text { split_Info }(C)
\end{aligned}
$$

Information gain ratio.

gain_ratio $(c)=I(B, C) / H(C)=$

$\operatorname{gain}(C) / \operatorname{split} \_\operatorname{Info}(C)$

\section{$6 \quad$ Classification Example}

The test data is the test results of small size bowls in a ceramics factory. The deformation caliber size error of a bowl is obtained by image processing technique. The crack and fall slag belong to the internal characteristics of bowls. These internal defects are extracted to get the defect data through the contrast increasement of linear gray value tensile. However, the harmonics and vibration of electronic devices lead to fuzzy image feature extraction so as to cause some great errors. These great errors are called outliers. In this paper, Kalman filtering algorithm is used to estimate test data tracks so as to eliminate abnormal data. Then, C4.5 decision tree algorithm is used to process the generalized data so as to get the entire test data tree. If the product test data meet the optimum conditions of the whole tree, the product is high quality product. If the product test data does not meet all optimal sub-tree layer conditions but at least meet the trunk tree conditions, then the product is qualified. If the product test data does not meet the above condition, the product is unqualified. The data processing flow chart is shown in Figure 10.

At first, taking deformation caliber size for example, raw data results of a batch of sample bowl deformation caliber sizes are shown in Table 1.

According to Chinese national standard, daily-use porcelain products within $\pm 1.5 \mathrm{~mm}$ caliber error are qualified. The standard caliber size of this kind of tested bowl is $60 \mathrm{~mm}$. The bowl caliber size errors are generally less than $3 \mathrm{~mm}$. However, from the table 1, we noticed that some data deviation is too large and can not appear in the actual production process. These data belong to abnormal data. Through using Kalman filtering algorithm, these abnormal data were filtered to get the data within error range which would be smoothed. Then, C4.5 decision tree was used to classify the bowl according to filtered data.

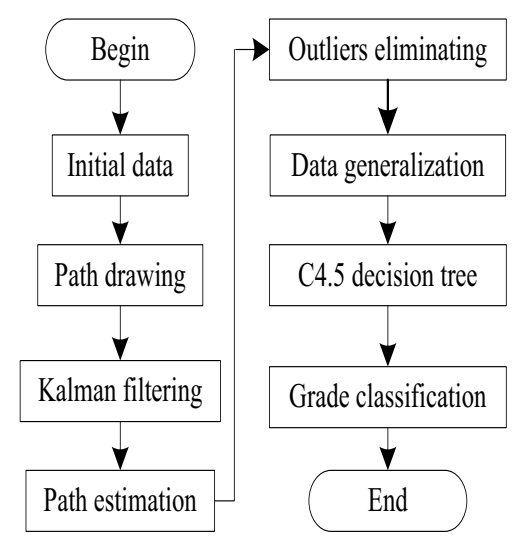

Figure 10. Data processing flow chart 
TABLE 1. SAMPLE INITIAL DATA

\begin{tabular}{|c|c|c|c|c|c|}
\hline Batch & \multicolumn{5}{|c|}{ Sample initial data } \\
\hline 1 & 60.4 & 60.3 & 61.2 & 61 & 60.1 \\
\hline 2 & 60 & 59.6 & 60.7 & 70 & 60.8 \\
\hline 3 & 59.3 & 61 & 62.1 & 67 & 60.6 \\
\hline 4 & 61.1 & 61.1 & 61.2 & 61.3 & 60.5 \\
\hline 5 & 59.8 & 69 & 60.8 & 60.9 & 60.2 \\
\hline
\end{tabular}

Secondly, these crack and fall flag defects are extracted to get the defect data through the contrast increasement of linear gray value tensile and utilization of Laplace point detection operator. In addition, through using image processing technique the boundary and image characteristics of roast flower sticky glaze are extracted and filtered to get test data sample error of relevant attributes. Table 2 is some defects test data error generalization table of some sample bowls. Due to the limited space, data acquisition process of Table 2 is omitted.

The application of $\mathrm{C} 4.5$ decision tree classification algorithm is as follows.

(1) Computing classification information about whether the product can pass the check of this class attribute, we can get the following results.

There are seven positive examples $y$ and seven counterexamples $\mathrm{n}$ about whether the product can pass the check.

$$
\begin{gathered}
P(y)=\frac{1}{2}, P(n)=\frac{1}{2}, \\
H(B)=-\frac{1}{2} \log _{2} \frac{1}{2}-\frac{1}{2} \log _{2} \frac{1}{2}=1
\end{gathered}
$$

TABLE 2. TEST DATA SAMPLE ERROR GENERALIZATION TABLE

\begin{tabular}{|c|c|c|c|c|c|}
\hline Sample & $\begin{array}{c}\text { Deformation } \\
\text { (Caliber mm })\end{array}$ & $\begin{array}{c}\text { Fall slag } \\
(\text { Diameter mm })\end{array}$ & $\begin{array}{c}\text { Crack } \\
(\text { Length mm })\end{array}$ & $\begin{array}{c}\text { Roast flower } \\
\text { sticky glaze } \\
\left(\text { Area mm }{ }^{2}\right.\end{array}$ & Pase checik \\
\hline 1 & $(0,1.0)$ & $(0.5,1.5)$ & $(0,3)$ & $\geqslant 5$ & Yse \\
\hline 2 & $(1,1.5)$ & $\geqslant 1.5$ & $\geqslant 6$ & $(0,1)$ & No \\
\hline 3 & $(0,1.0)$ & $(0,0.5)$ & $(0,3)$ & $(1,5)$ & Yse \\
\hline 4 & $(0,1.0)$ & $(0.5,1.5)$ & $(3,6)$ & $\geqslant 5$ & No \\
\hline 5 & $(1,1.5)$ & $(0,0.5)$ & $(0,3)$ & $\geqslant 5$ & Yse \\
\hline 6 & $(1,1.5)$ & $(0.5,1.5)$ & $(3,6)$ & $(0,1)$ & Yse \\
\hline 7 & $\geqslant 1.5$ & $(0.5,1.5)$ & $(3,6)$ & $(0,1)$ & No \\
\hline 8 & $(0,1.0)$ & $(0,0.5)$ & $(0,3)$ & $(1,5)$ & No \\
\hline 9 & $(1,1.5)$ & $(0,0.5)$ & $(0,3)$ & $(1,5)$ & Yse \\
\hline 10 & $(0,1.0)$ & $(0.5,1.5)$ & $(3,6)$ & $(1,5)$ & Yse \\
\hline 11 & $(1,1.5)$ & $\geqslant 1.5$ & $(0,3)$ & $(0,1)$ & Yse \\
\hline 12 & $\geqslant 1.5$ & $\geqslant 1.5$ & $(3,6)$ & $(0,1)$ & No \\
\hline 13 & $(0,1.0)$ & $(0.5,1.5)$ & $\geqslant 6$ & $(0,1)$ & No \\
\hline 14 & $\geqslant 1.5$ & $(0,0.5)$ & $\geqslant 6$ & $(1,5)$ & No \\
\hline
\end{tabular}

(2) Taking "deformation attribute" as an example, there are different kind of caliber bowls which are with $(0,1.0)$, $(1,1.5)$ and $\geq 1.5$ grade in Table 2 . The number of bowls with
$(0,1.0) \mathrm{mm},(1,1.5)$ and $\geq 1.5$ grade is 6,5 and 3 respectively. When the bowl caliber size error is in $(0.1 .0) \mathrm{mm}$, $y=3, n=3$; When the bowl caliber size error is in $(1,1.5) \mathrm{mm}, y=4, n=1$; When the bowl caliber size error is greater than or equal to $1.5, y=0, n=3$. According to equations (12)-(16), we get

$$
\begin{gathered}
H(B / C)=-\frac{6}{14}\left(\frac{1}{2} \log _{2} \frac{1}{2}+\frac{1}{2} \log _{2} \frac{1}{2}\right)-\frac{5}{14}\left(\frac{4}{5} \log _{2} \frac{4}{5}+\right. \\
\left.\quad \frac{1}{5} \log _{2} \frac{1}{5}\right)-\frac{3}{14}\left(0 \log _{2} 0+1 \log _{2} 1\right)=0.69 \\
H(C)=-\left(\frac{6}{14} \log _{2} \frac{6}{14}+\frac{5}{14} \log _{2} \frac{5}{14}+\frac{3}{14} \log _{2} \frac{3}{14}\right)=1.52 \\
\text { gain_ratio }(c)=I(B, C) / H(C)=\frac{1-0.69}{1.52}=0.20
\end{gathered}
$$

(3) Similarly, information gain rate of "fall flag", "crack", and " roast flower sticky glaze" is as following respectively.

Fall slag gain_ratio $(c)=I(B, C) / H(C)=\frac{1-0.97}{1.1}=0.03$

Crack gain_ratio $(c)=I(B, C) / H(C)=\frac{1-0.63}{1.1}=0.34$

Roast flower sticky glaze

$$
\text { gain_ratio }(c)=I(B, C) / H(C)=\frac{1-0.97}{1.43}=0.05
$$

Through comparing the gain rate of each attribute, it is known that the information gain of crack is the largest in this example. Hence, the crack is selected as the root node test attribute. The root node of the defect attribute is shown in Figure 11.

Comparing the condition information entropy of each attribute by calculating, the sub-trees are established successively as shown in Figure 12.and Figure 13.

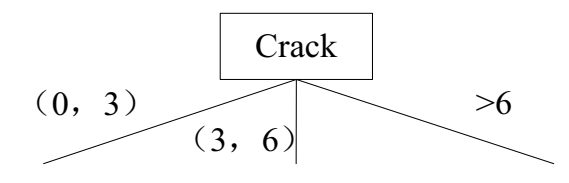

Figure 11. The decision tree step 1

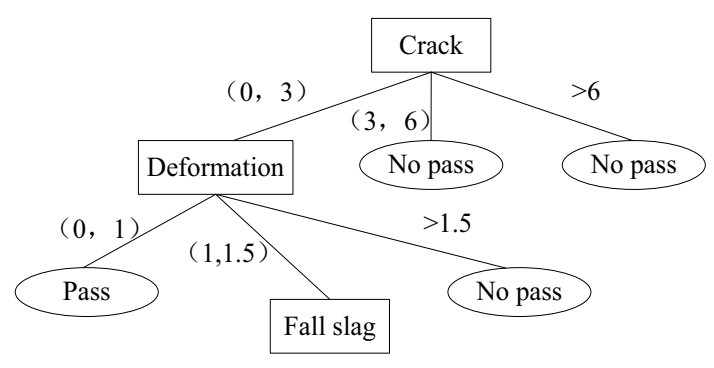

Figure 12. The decision tree step 2 


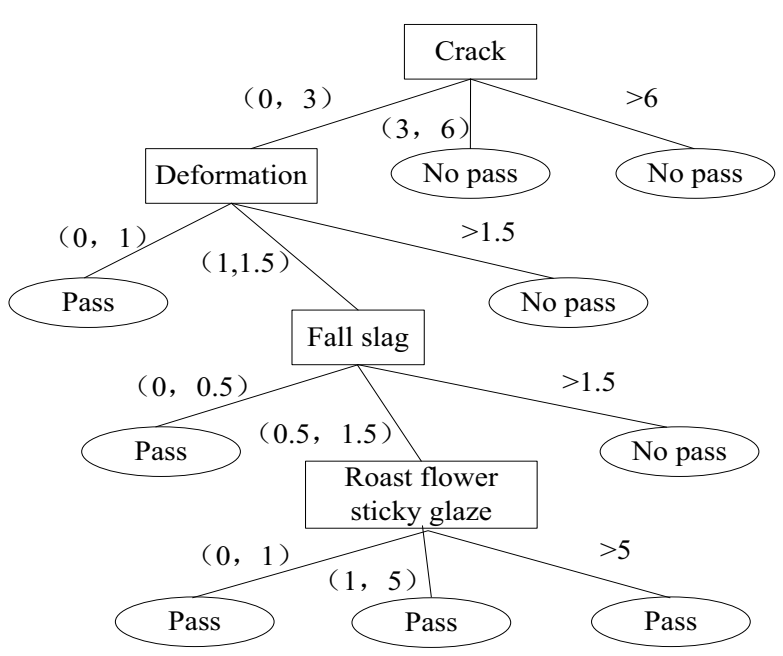

Figure 13. The decision tree step 3

For example, the part of the classification rules is as follows.

(1) If crack length is less than $3 \mathrm{~mm}$ and deformation caliber size error is less than $1 \mathrm{~mm}$, then the check is passed.

(2) If crack length is less than $3 \mathrm{~mm}$ and deformation caliber size error is within $(1,1.5) \mathrm{mm}$ and fall slag diameter is less than $0.5 \mathrm{~mm}$, then the check is passed.

(3) If crack length is Is greater than $3 \mathrm{~mm}$, then the check is not passed.

(4) If crack length is less than $3 \mathrm{~mm}$ and deformation caliber error is greater than $1.5 \mathrm{~mm}$, then the check is not passed.

(5) If crack length is less than $3 \mathrm{~mm}$ and deformation caliber error is within $(1,1.5) \mathrm{mm}$ and fall slag diameter is greater than $0.5 \mathrm{~mm}$, then the check is not passed.

By comparing the gain rate of each attribute, we can know that the crack is chosen as the test attribute. Then, a decision tree is established to obtain the next level of test attributes until all attributes are tested through using recursive method. At last, according to "If ... and ....and..., then..." rule, the test results are determined and the porcelain quality grade classification work has be finished.

\section{Summary}

In the paper, multi-agent technology-based daily-use porcelain detection and quality grade classification method had been studied. A small alliance type multi-agent network was established to detect and to classify daily-use porcelain products based on the complete multi-agent network structure of the whole ceramic production process control system. Based on discrete Kalman filtering algorithm and C4.5 decision tree classification algorithm, the classification algorithm used for daily-use porcelain product is proposed. Multi-agent-based detection and classification system structure of daily-use porcelain quality and the implementation of various functions are presented. Finally, an application example is given and demonstrates the feasibility and effectiveness of the method proposed. This will provide a new idea and new method for the detection and classification of daily-use porcelain quality and expand the application fields of multi-agent technology. Hence, the method proposed in the paper is with important application value. In the future, this method will be further optimized so as to be able to be applied in the practical engineering.

\section{Acknowledgement}

This work was financially supported by the National Natural Science Foundation of China (No. 61563022 and No. 61402209) and Jiangxi Province Natural Science Foundation of China (No. 20152ACB20009).

\section{References}

[1] G. Q. Zhong, G. L. Hu, and Z. X. Zhang, "Defect analysis for sanitary ceramics," China Ceramic Industry(In Chinese),. Vol.15, pp.35-37 2008.

[2] L.L. Li, C.Z. Gong, and K.J. Zhu, "Multi-agent simulation on natural gas time-of-use pricing in urban industrial and commercial users, " Systems Engineering Theory \& Practice(In Chinese),vol. 34, pp. 392398.,2014

[3] X.M. Luo, "Research on the combat efficiency evaluation modeling of anti-radiation missile based on multi-agent technique," Journal of the Academy of Equipment Command\&Technology(In Chinese). vol. 21, pp. 111-115, 2010.

[4] W.N. Xie, P.A. Qiao, and X.Y. Pan, "Change detection in SAR images using multi-agent genetic and twodimensional Otsu algorithm," Computer Engineering and Design(In Chinese), vol. 35, pp. 3532- 3537, 2014.

[5] J. H. Lin, Z. Liu, and Y.F. Chen, "An emotion decision-making model in multi-agent environment," PR\&AI (In Chinese), vol. 28, pp. 369-376, 2015.

[6] W.K.Liu, "Study on cooperative control and optimization of complex multi-agent networks[D]," Wuhan: Huazhong University of Science \&Technology (In Chinese), pp. 6-19, 2011.

[7] Y.H. Guo, G. Goncalves, and T. Hsu, "Multi-agent Based Self-adaptive Genetic Algorithm for the Longterm Car Pooling Problem, “ Journal of Mathematical Modelling and Algorithms in Operations Research, vol. 20, pp. 45-66, 2013.

[8] G.Y. Sato, H.J.S.D. Azevedo, and J.P.A. Barthès, "Agent and multi-agent applications to support distributed communities of practice: a short review," Autonomous Agents and Multi-Agent Systems. Vol. 25, pp. 87-129, 2012.

[9] A. Birukou, E. Blanzieri, and P. Giorgini, "Implicit: a multi-agent recommendation system for web search," 
Autonomous Agents and Multi-Agent Systems, vol. 24, pp. 141-174, 2012

[10] F.M.A. Klingert, and M. Meyer, "Effectively combining experimental economics and multi-agent simulation: suggestions for a procedural integration with an example from prediction markets research," Computational \& Mathematical Organization Theory, vol. 18, pp. 63-90, 2012. 